\title{
Curve/Surface Intersection Problem by means of Matrix Representations
}

\author{
Thang Luu Ba, Laurent Busé, Bernard Mourrain \\ GALAAD, INRIA \& UMR CNRS 6621,UNSA \\ BP 93, 06902 Sophia-Antipolis, France
}

\begin{abstract}
In this paper, we introduce matrix representations of algebraic curves and surfaces for Computer Aided Geometric Design (CAGD). The idea of using matrix representations in CAGD is quite old. The novelty of our contribution is to enable non square matrices, extension which is motivated by recent research in this topic. We show how to manipulate these representations by proposing a dedicated algorithm to address the curve/surface intersection problem by means of numerical linear algebra techniques.
\end{abstract}

\section{Introduction}

Computing the intersection between algebraic varieties is a fundamental task in Computer Aided Geometric Design. Several methods and approaches has been developed for that purpose. Some of them are based on matrix representations of the objects that allows to transform the computation of the intersection locus into generalized eigencomputations (see for instance [16, 1, 9] and the references therein). As far as we know, all of these methods have only been developed with square matrix representation. The aim of this paper is to show that similar algorithms can be implemented even if the matrix representation used are non square matrices. Notice that recent researches, known under the name of the moving surfaces method, have demonstrated that these non square representation matrices are much more easy to compute than square representation matrices. Moreover, they appear under much less restrictive hypothesis, notably regarding so-called base points.

The approach to the curve/surface intersection problem we will develop in the sequel consists in two main steps. The first one is the computation of a matrix representation of the surface from its parametrization. After mixing this matrix representation of the surface with the parameterization of the curve, the second step consists of a matrix reduction then eigencomputation. As a particularity of our method, these two steps can be performed either by symbolic exact computations or by numerical computation, based on classical numerical 
linear algebra tools. However, a good combination seems to be the symbolic treatment for the first step related to the moving plane computation, so that the change of representation does not affect the intersection locus, and then numerical computation, typically LU-decomposition and eigenvalues computation, to end the algorithm.

The paper is organized as follows. In Section 2, we define what is a representation matrix of a parametrized surface. In Section 3, we introduce the intersection problem and explain how to use generalized eigenvalues for computing the intersection points. We also present the method, based on LU-decomposition, that allows us to extract the regular part of a Kronecker form of a pencil of matrices. In Section 4, we analyze the multiplicity of an intersection point. Finally, we give some examples and suggest future researches in Section 5 and Section 6.

\section{Matrix based implicit representations}

Hereafter, $\mathbb{K}$ denotes an infinite field.

Given a parametrized algebraic surface, the aim of this section is to build a matrix that represents this surface in a way that we will make explicit. The entries of this matrix are linear in the space of implicit variables. In order to clarify our approach and put it in perspective, we begin with the more simple case of parametrized algebraic plane curves.

\subsection{Rational plane algebraic curves}

Suppose given a parametrization

$$
\begin{aligned}
\mathbb{P}_{\mathbb{K}}^{1} & \stackrel{\phi}{\rightarrow} \mathbb{P}_{\mathbb{K}}^{2} \\
(s: t) & \mapsto\left(f_{1}: f_{2}: f_{3}\right)(s: t)
\end{aligned}
$$

of a plane algebraic curve $\mathcal{C}$ in $\mathbb{P}^{2}$. We set $d:=\operatorname{deg}\left(f_{i}\right) \geq 1, i=1,2,3$ and denote by $x, y, z$ the homogeneous coordinates of the projective plane $\mathbb{P}_{\mathbb{K}}^{2}$. The implicit equation of $\mathcal{C}$ is a homogeneous polynomial $C \in \mathbb{K}[x, y, z]$ satisfying the property $C\left(f_{1}, f_{2}, f_{3}\right) \equiv 0$ and with the smallest possible degree (notice that $C$ is actually defined up to multiplication by a nonzero element of $\mathbb{K}$ ). It is well known that

$$
\operatorname{deg}(\phi) \operatorname{deg}(C)=d-\operatorname{deg}\left(\operatorname{gcd}\left(f_{1}, f_{2}, f_{3}\right)\right)
$$

where $\operatorname{deg}(\phi)$ is the degree of the parametrization $\phi$. Roughly speaking, the integer $\operatorname{deg}(\phi)$ measures the number of times the curve $\mathcal{C}$ is drawn by the parametrization $\phi$. For simplicity, from now on we will assume that

$$
\operatorname{gcd}\left(f_{1}, f_{2}, f_{3}\right) \in \mathbb{K} \backslash\{0\},
$$

that is to say that the parametrization $\phi$ is defined everywhere. 
We can build a collection of matrices that are associated to the parametrization $\phi$ as follows. For all non negative integer $\nu$, consider the set $\mathcal{L}_{\nu}$ of polynomials of the form

$$
a_{1}(s, t) x+a_{2}(s, t) y+a_{3}(s, t) z \in \mathbb{K}[s, t][x, y, z]
$$

such that

- $a_{i}(s, t) \in \mathbb{K}[s, t]$ is homogeneous of degree $\nu$ for all $i=1,2,3$ and

- $\sum_{i=1}^{3} a_{i}(s, t) f_{i}(s, t) \equiv 0$ in $\mathbb{K}[s, t]$.

By definition, it is clear that $\mathcal{L}_{\nu}$ is a $\mathbb{K}$-vector space and that a basis, say $L^{(1)}, \ldots, L^{\left(n_{\nu}\right)}$, of $\mathcal{L}_{\nu}$ can be computed by solving a single linear system with indeterminates the coefficients of the polynomials $a_{i}(s, t), i=1,2,3$. The matrix $\mathrm{M}(\mathbf{f})_{\nu}$ is the matrix of coefficients of $L^{(1)}, \ldots, L^{\left(n_{\nu}\right)}$ as homogeneous polynomials of degree $\nu$ in the variables $s, t$. In other words, we have the equality

$$
\left[\begin{array}{llll}
s^{\nu} & s^{\nu-1} t & \cdots & t^{\nu}
\end{array}\right] \mathrm{M}(\mathbf{f})_{\nu}=\left[\begin{array}{llll}
L^{(1)} & L^{(2)} & \cdots & L^{\left(n_{\nu}\right)}
\end{array}\right]
$$

The entries of $\mathrm{M}(\mathbf{f})_{\nu}$ are linear forms in $\mathbb{K}[x, y, z]$. As the integer $\nu$ varies, we have the following picture for the size of the matrix $\mathrm{M}(\mathbf{f})_{\nu}$ :

- if $0 \leq \nu \leq d-2$ the number $n_{\nu}$ of columns is strictly less than $\nu+1$ which is the number of rows,

- if $\nu=d-1$ then $\mathrm{M}(\mathbf{f})_{d-1}$ is a square matrix of size $d$,

- if $\nu \geq d$ the number $n_{\nu}$ of columns is strictly bigger than $\nu+1$ which is the number of rows.

Proposition $1([8])$ For all $\nu \geq d-1$ the two following properties hold :

- the GCD of the minors of (maximum) size $\nu+1$ of $\mathrm{M}(\mathbf{f})_{\nu}$ is equal to $C(x, y, z)^{\operatorname{deg}(\phi)}$ up to multiplication by a nonzero element in $\mathbb{K}$,

- $\mathrm{M}(\mathbf{f})_{\nu}$ is generically full rank and its rank drops exactly on the curve $\mathcal{C}$.

This result shows that all the matrices $\mathrm{M}(\mathbf{f})_{\nu}$ such that $\nu \geq d-1$, can serve as an implicit representation of the curve $\mathcal{C}$ in the same way as the implicit equation $C(x, y, z)$ is an implicit representation of the curve $\mathcal{C}$.

The matrix $\mathrm{M}(\mathbf{f})_{d-1}$ is particularly interesting because it is the smallest matrix representing the curve $\mathcal{C}$ and especially because it is a square matrix, which implies that

$$
\operatorname{det}\left(\mathrm{M}(\mathbf{f})_{d-1}\right)=c \cdot C(x, y, z)^{\operatorname{deg}(\phi)}
$$

where $c \in \mathbb{K} \backslash\{0\}$. This matrix goes back, as far as we know, to the work [19] and has been widely exploited since then by the community of Geometric Modeling and Computer Aided Geometric Design as the method of moving lines.

It is natural to wonder if such an approach can be carried out to the case of parametrized algebraic surfaces. As we will see, most of the above results 
hold in this case with much more involved details and some suitable hypothesis. However, it turns out that a matrix similar to the matrix $\mathrm{M}(\mathbf{f})_{d-1}$ rarely exists. Therefore, in order to keep a square matrix it is necessary to introduce quadratic syzygies, or higher order syzygies; see for instance $[10,6,14]$. In the sequel, we will stick to the case of linear syzygies because of their simplicity and generality, even if we will not get square matrices in general.

\subsection{Rational algebraic surfaces}

Suppose given a parametrization

$$
\begin{aligned}
\mathbb{P}_{\mathbb{K}}^{2} & \stackrel{\phi}{\longrightarrow} \mathbb{P}_{\mathbb{K}}^{3} \\
(s: t: u) & \mapsto\left(f_{1}: f_{2}: f_{3}: f_{4}\right)(s, t, u)
\end{aligned}
$$

of a surface $\mathcal{S}$ such that $\operatorname{gcd}\left(f_{1}, \ldots, f_{4}\right) \in \mathbb{K} \backslash\{0\}$. Set $d:=\operatorname{deg}\left(f_{i}\right) \geq 1$, $i=1,2,3,4$ and denote by $S(x, y, z, w) \in \mathbb{K}[x, y, z, w]$ the implicit equation of $\mathcal{S}$ which is defined up to multiplication by a nonzero element in $\mathbb{K}$. Similarly to the case of parametrized plane curves, there also exists a degree formula that asserts that the quantity $\operatorname{deg}(\mathcal{S}) \operatorname{deg}(\phi)$ is equal to $d^{2}$ minus the number of common roots of $f_{1}, f_{2}, f_{3}, f_{4}$ in $\mathbb{P}^{2}$ counted with suitable multiplicities (see for instance [8, Theorem 2.5] for more details).

We build a collection of matrices associated to the parametrization $\phi$ as follows. For all non negative integer $\nu$, consider the set $\mathcal{L}_{\nu}$ of polynomials of the form

$$
a_{1}(s, t, u) x+a_{2}(s, t, u) y+a_{3}(s, t, u) z+a_{4}(s, t, u) w
$$

such that

- $a_{i}(s, t, u) \in \mathbb{K}[s, t, u]$ is homogeneous of degree $\nu$ for all $i=1, \ldots, 4$,

- $\sum_{i=1}^{4} a_{i}(s, t, u) f_{i}(s, t, u) \equiv 0$ in $\mathbb{K}[s, t, u]$.

This set is a $\mathbb{K}$-vector space; denote by $L^{(1)}, \ldots, L^{\left(n_{\nu}\right)}$ a basis of it that can be computed by solving a single linear system. Then, define the matrix $M(f)_{\nu}$ by the equality

$$
\left[\begin{array}{llll}
s^{\nu} & s^{\nu-1} t & \cdots & u^{\nu}
\end{array}\right] \mathrm{M}(\mathbf{f})_{\nu}=\left[\begin{array}{llll}
L^{(1)} & L^{(2)} & \cdots & L^{\left(n_{\nu}\right)}
\end{array}\right]
$$

Before giving the main properties of this collection of matrices, we need the following

Definition 1 A matrix $\mathrm{M}(\mathbf{f})$ with entries in $\mathbb{K}[x, y, z, w]$ is said to be a representation of a given homogeneous polynomial $P \in \mathbb{K}[x, y, z, w]$ if

i) $\mathrm{M}(\mathbf{f})$ is generically full rank,

ii) the rank of $\mathrm{M}(\mathbf{f})$ drops exactly on the surface of equation $P=0$,

ii) the GCD of the maximal minors of $\mathrm{M}(\mathbf{f})$ is equal to $P$, up to multiplication by a nonzero constant in $\mathbb{K}$. 
Recall that a point in $\mathbb{P}_{\mathbb{K}}^{2}$ is called a base point of the parametrization $\phi$ if it is a common root of the polynomials $f_{1}, \ldots, f_{4}$. It is said to be locally a complete intersection if it can be locally generated by two equations, and said to be locally an almost complete intersection if it can be locally generated by three equations.

Proposition $2([4,5])$ For all integer $\nu \geq 2(d-1)$ we have:

- if the base points are local complete intersections then $\mathrm{M}(\mathbf{f})_{\nu}$ represents $S^{\operatorname{deg}(\phi)}$,

- if the base points are almost local complete intersections then $\mathrm{M}(\mathbf{f})_{\nu}$ represents

$$
S^{\operatorname{deg}(\phi)} \times \prod_{\mathfrak{p} \in V\left(f_{1}, \ldots, f_{4}\right) \subset \mathbb{P}_{\mathbb{K}}^{2}} L_{\mathfrak{p}}(x, y, z, w)^{e_{\mathfrak{p}}-d_{\mathfrak{p}}}
$$

where $L_{\mathfrak{p}}(x, y, z, w)$ are linear forms.

Remark 1 It is possible to improve the bound $2(d-1)$ by taking into account the geometry of the base points; we refer the reader to [4] for more details. For instance, if there exists at least one common root to $f_{1}, \ldots, f_{4}$ in $\mathbb{P}^{2}$ then the above proposition is true for all $\nu \geq 2(d-1)-1$. Also, mentioned that the linear forms $L_{\mathfrak{p}}(x, \ldots, w)$ can be determined by computations of syzygies in $\mathbb{K}[s, t, u]$; see [5].

Although we are dealing with surfaces parametrized by the projective plane, it is important to mention that the above results still hold for surfaces parametrized by the product of two projective lines, or more generally by a toric variety. We refer the interested reader to [7] and [3] for these extensions.

\section{Curve/surface intersection}

From now on, and until the end of the paper, we assume that $\mathbb{K}$ is an algebraically closed field, typically the field of complex numbers $\mathbb{C}$.

Suppose given an algebraic surface $\mathbf{S}$ represented by a homogeneous and irreducible implicit equation $S(x, y, z, w)=0$ in $\mathbb{P}_{\mathbb{K}}^{3}$ and a rational space curve C represented by a parameterization

$$
\Psi: \mathbb{P}_{\mathbb{K}}^{1} \rightarrow \mathbb{P}_{\mathbb{K}}^{3}:(s: t) \mapsto(x(s, t): y(s, t): z(s, t): w(s, t))
$$

where $x(s, t), y(s, t), z(s, t), w(s, t)$ are homogeneous polynomials of the same degree and without common factor in $\mathbb{K}[s, t]$.

A standard problem in non linear computational geometry is to determine the set $\mathbf{C} \cap \mathbf{S} \subset \mathbb{P}_{\mathbb{K}}^{3}$, especially when it is finite. One way to proceed, is to compute the roots of the homogeneous polynomial

$$
S(x(s, t), y(s, t), z(s, t), w(s, t))
$$


because they are in correspondence with $\mathbf{C} \cap \mathbf{S}$ through the regular map $\Psi$. Observe that (1) is identically zero if and only if $\mathbf{C} \cap \mathbf{S}$ is infinite, equivalently $\mathbf{C} \subset \mathbf{S}$ (for $\mathbf{C}$ is irreducible).

If $\mathbf{S}$ is a rational surface represented by a parameterization, then several authors (see for instance [16] and the references therein) used some square matrix representations, most of the time obtained from a particular resultant matrix, of $\mathbf{S}$ in order to compute the set $\mathbf{C} \cap \mathbf{S}$ by means of eigencomputations. As we have already mentioned, such square matrix representations exist only under some restrictive conditions. Hereafter, we would like to generalize this approach for non square matrix representation that can be obtained for a much larger class of rational surfaces and are very easy to compute.

So, assume that $M(x, y, z, w)$ is a matrix representation of the surface $\mathbf{S}$, meaning a representation of the polynomial $S(x, y, z, w)$. By replacing the variables $x, y, z, w$ by the homogeneous polynomials $x(s, t), y(s, t), z(s, t), w(s, t)$ respectively, we get the matrix

$$
M(s, t)=M(x(s, t), y(s, t), z(s, t), w(s, t))
$$

and we have the following easy property:

Lemma 1 With the above notation, for all point $\left(s_{0}: t_{0}\right) \in \mathbb{P}_{\mathbb{K}}^{1}$ the rank of the matrix $M\left(s_{0}, t_{0}\right)$ drops if and only if the point $\left(x\left(s_{0}, t_{0}\right): y\left(s_{0}, t_{0}\right): z\left(s_{0}, t_{0}\right)\right.$ : $\left.w\left(s_{0}, t_{0}\right)\right)$ belongs to the intersection locus $\mathbf{C} \cap \mathbf{S}$.

It follows that points in $\mathbf{C} \cap \mathbf{S}$ associated to points $(s: t)$ such that $s \neq 0$, are in correspondence with the set of values $t \in \mathbb{K}$ such that $M(1, t)$ drops of rank strictly less than its row and column dimensions.

In what follows, we will develop a numerical method to reduce generalized pencils of matrices. More precisely, in the theory of Kronecker forms (see for instance [12, Chapitre 12]) we will reduce such a pencil to its regular part, avoiding this way the non square Kronecker blocks.

\subsection{Linearization of a polynomial matrix}

We begin with some notation.

Let $A$ and $B$ be two matrices of size $m \times n$. We will call a generalized eigenvalue of $\mathrm{A}$ and $\mathrm{B}$ a value in the set

$$
\lambda(A, B):=\{t \in \mathbb{K}: \operatorname{rank}(A-t B)<\min \{m, n\}\}
$$

In the case $m=n$, the matrices $\mathrm{A}$ and $\mathrm{B}$ have $n$ generalized eigenvalues if and only if $\operatorname{rank}(B)=n$. If $\operatorname{rank}(B)<n$, then $\lambda(A, B)$ can be finite, empty or infinite. Moreover, if $\mathrm{B}$ is invertible then $\lambda(A, B)=t\left(A B^{-1}, I\right)=t\left(A B^{-1}\right)$, which is the ordinary spectrum of $A B^{-1}$. The previous definition of generalized eigenvalues extends naturally to a polynomial matrix $M(t)$, where the entries are polynomials in $t$ of any degree. 
Suppose given an $m \times n$-matrix $M(t)=\left(a_{i, j}(t)\right)$ with polynomial entries $a_{i, j}(t) \in \mathbb{K}[t]$. It can be equivalently written as a polynomial in $t$ with coefficients $m \times n$-matrices with entries in $\mathbb{K}$ : if $d=\max _{i, j}\left\{\operatorname{deg}\left(a_{i, j}(t)\right)\right\}$ then

$$
M(t)=M_{d} t^{d}+M_{d-1} t^{d-1}+\ldots+M_{0}
$$

where $M_{i} \in \mathbb{K}^{m \times n}$.

Definition 2 The generalized companion matrices $A, B$ of the matrix $M(t)$ are the matrices with coefficients in $\mathbb{K}$ of size $((d-1) m+n) \times d m$ that are given by

$$
\begin{gathered}
A=\left(\begin{array}{ccccc}
0 & I & \ldots & \ldots & 0 \\
0 & 0 & I & \ldots & 0 \\
\vdots & \vdots & \vdots & \vdots & \vdots \\
0 & 0 & \ldots & \ldots & I \\
M_{0}^{t} & M_{1}^{t} & \ldots & \ldots & M_{d-1}^{t}
\end{array}\right) \\
B=\left(\begin{array}{ccccc}
I & 0 & \ldots & \ldots & 0 \\
0 & I & 0 & \ldots & 0 \\
\vdots & \vdots & \vdots & \vdots & \vdots \\
0 & 0 & \ldots & I & 0 \\
0 & 0 & \ldots & \ldots & -M_{d}^{t}
\end{array}\right)
\end{gathered}
$$

where I stands for the identity matrix and $M_{i}^{t}$ stands for the transpose of the matrix $M_{i}$.

We have the following interesting property that follows from a straightforward computation.

Proposition 3 With the above notation, for all $t \in \mathbb{K}$ and all vector $v \in \mathbb{K}^{m}$ we have

$$
M^{t}(t) v=0 \Leftrightarrow(A-t B)\left(\begin{array}{c}
v \\
t v \\
\vdots \\
t^{d-1} v
\end{array}\right)=0 .
$$

Because rank $M(t)=\operatorname{rank} M^{t}(t)$, from now on we will assume that $M(t)$ is an $m \times n$-matrix such that $m \leq n$. Therefore, $\operatorname{rank} M(t)$ drops if and only if $\operatorname{rank} M(t)<m$.

Theorem 1 With the above assumptions, the following equivalence holds:

$$
\operatorname{rank} M(t)<m \Leftrightarrow \operatorname{rank}(A-t B)<d m .
$$

Proof. Because rank $M(t)=\operatorname{rank} M^{t}(t)$, we have that $\operatorname{rank} M^{t}(t)<m$. Thus, there exists a column vector $v \neq 0$ such that $M^{t}(t) v=0$. Then, by Proposition 3 equation $(A-t B) x=0$ has a nonzero root. That means exactly that $\operatorname{rank}(A-$ $t B)<d m$. 
Now, if $\operatorname{rank}(A-t B)<d m$, then equation $(A-t B) x=0$ have a root $x \neq 0$ and by a straightforward computation it is of the form

$$
x=\left(\begin{array}{c}
v \\
t v \\
\vdots \\
t^{d-1} v
\end{array}\right)
$$

Since $x \neq 0$ and by Proposition 3, we have $v \neq 0$ and $\mathrm{v}$ is a root of equation $M^{t}(t) v=0$. Thus, $\operatorname{rank} M^{t}(t)<m$ and it follows that $\operatorname{rank} M(t)<m$.

By Theorem 1, we transformed the computation of generalized eigenvalues of the matrix polynomial $M(t)$ (that is to say the roots of the gcd of the maximal minors of $M(t))$ into the computation of generalized eigenvalues of a pencil of matrices $A-t B$. If the matrices $A, B$ were two square matrices, then we could easily compute their generalized eigenvalues by the QZ-algorithm [13]. Therefore, our next task is to reduce the pencil $A-t B$ into a square pencil that keeps the information we are interested in.

Before moving on, we recall what is the Smith form of $M(t)$ for future use. Assume that $\operatorname{rank} M(t)=r$, it exists two regular polynomial matrices with nonzero determinant in $\mathbb{K}$, say $P(t)$ and $Q(t)$, such that

$$
D(t)=P(t) M(t) Q(t)=\left(\begin{array}{cccccc}
a_{r}(t) & 0 & \ldots & \ldots & \ldots & 0 \\
0 & a_{r-1}(t) & 0 & \ldots & \ldots & 0 \\
\vdots & \vdots & \vdots & \vdots & \vdots & \vdots \\
0 & \ldots & \ldots & a_{1}(t) & \ldots & 0 \\
0 & 0 & \ldots & \ldots & \ldots & 0
\end{array}\right)
$$

where $a_{i}(t)$ 's are monic polynomials and $a_{i}(t)$ divides $a_{i-1}(t)$. This form is unique and is called the Smith form of $M(t)$ (see for instance [11, Chapter 6]). Notice that by performing unimodular row and column transformations on the matrix $A-t B$, we can find that $A-t B$ has the Smith form (see for instance [20] for more details)

$$
U(t)(A-t B) V(t)=\operatorname{diag}\left\{I_{m}, \ldots, I_{m}, D(t)\right\}
$$

where $D(t)$ is the Smith form of $M^{t}(t)$. Thus, Theorem 1 can be recovered from this property.

\subsection{The Kronecker form of a non square pencil of matrices}

Hereafter, we recall some known properties of the Kronecker form of pencils of matrices.

Definition 3 Let $L_{k}(t), \Omega_{k}(t)$ be the two matrices of size $k \times(k+1)$ and $k \times k$ 
respectively, defined by

$$
\begin{aligned}
L_{k}(t) & =\left(\begin{array}{ccccc}
1 & t & 0 & \ldots & 0 \\
0 & 1 & t & \ldots & 0 \\
\vdots & \vdots & \vdots & \vdots & \vdots \\
0 & \ldots & 1 & t & 0 \\
0 & 0 & \ldots & 1 & t
\end{array}\right), \\
\Omega_{k}(t) & =\left(\begin{array}{ccccc}
1 & t & 0 & \ldots & 0 \\
0 & 1 & t & \ldots & 0 \\
\vdots & \vdots & \vdots & \vdots & \vdots \\
0 & \ldots & \ldots & 1 & t \\
0 & 0 & \ldots & 0 & 1
\end{array}\right) .
\end{aligned}
$$

We are going to use the following theorem, which gives what is called the Kronecker canonical form of a pencil of matrices (see for instance [12, p. 31-34]).

Theorem 2 For any couple constant matrices $A, B$ of size $p \times q$, there exist constant invertible matrices $P$ and $Q$ such that the pencil $P(A-t B) Q$ is of the block-diagonal form

$$
\operatorname{diag}\left\{L_{i_{1}}, \ldots, L_{i_{s}}, L_{j_{1}}^{t}, \ldots, L_{j_{u}}^{t}, \Omega_{k_{1}}, \ldots, \Omega_{k_{v}}, A^{\prime}-t B^{\prime}\right\}
$$

where $A^{\prime}, B^{\prime}$ are square matrices and $B^{\prime}$ is invertible. The dimensions $i_{1}, \ldots, i_{s}$, $j_{1}, \ldots, j_{u}, k_{1}, . ., k_{v}$ and the determinant of $A^{\prime}-t B^{\prime}$ (up to a scalar) are independent of the representation.

This theorem can be implemented as follows:

Proposition 4 For any couple of matrices $C_{0}, C_{1}$ of size $p \times q$, there exist unitary matrices $U$ and $V$ such that the pencil

$$
U\left(C_{0}-t C_{1}\right) V=\tilde{C}_{0}-t \tilde{C}_{1}
$$

is of the form

$$
\tilde{C}(t)=\left(\begin{array}{ccc}
\tilde{C}_{l}(t) & \tilde{C}_{1,2}(t) & \tilde{C}_{1,3}(t) \\
0 & \tilde{C}_{r}(t) & \tilde{C}_{2,2}(t) \\
0 & 0 & \tilde{C}_{r e g}(t)
\end{array}\right)
$$

where

- $\tilde{C}_{l}(t)=\tilde{C}_{l, 0}-t \tilde{C}_{l, 1}$ has only blocks of the form $L_{k}(t), \Omega_{k}(t)$ in its Kronecker canonical form,

- $\tilde{C}_{r}(t)=\tilde{C}_{r, 0}-t \tilde{C}_{r, 1}$ has only blocks of the form $L_{k}^{t}(t)$,

- $\tilde{C}_{r e g}(t)=\tilde{C}_{r e g, 0}-t \tilde{C}_{r e g, 1}$ is a square regular pencil. 
It is interesting to notice that the above decomposition can be computed within $O\left(p^{2} q\right)$ arithmetic operations. We refer the reader to [2] for a proof, as well as for an analysis of the stability of this decomposition.

Following the ideas developed in [2] and the reduction methods exploited in $[17,18]$, we now describe an algorithm that allows to remove the Kronecker blocks $L_{k}, L_{k}^{t}$ and $\Omega_{k}$ of the pencil of matrices $A-t B$ in order to extract the regular pencil $A^{\prime}-t B^{\prime}$.

\subsection{The Algorithm for extracting the regular part of a non square pencil of matrices}

We start with a pencil $A-t B$ where $A, B$ are constant matrices of size $p \times q$. Set $\rho=\operatorname{rank} B$. In the following algorithm, all computational steps are easily realized via the classical LU-decomposition.

Step 1 Transform $B$ into its column echelon form; that amounts to determine unitary matrices $P_{0}$ and $Q_{0}$ such that

$$
B_{1}=P_{0} B Q_{0}=[\underbrace{B_{1,1}}_{\rho} \mid \underbrace{0}_{q-\rho}]
$$

where $B_{1,1}$ is an echelon matrix. Then, compute

$$
A_{1}=P_{0} A Q_{0}=[\underbrace{A_{1,2}}_{\rho} \mid \underbrace{A_{1,2}}_{q-\rho}]
$$

Step 2 Transform $A_{1,2}$ into its row echelon form; that amounts to determine unitary matrices $P_{1}$ and $Q_{1}$ such that

$$
P_{1} A_{1,2} Q_{1}=\left(\frac{A_{1,2}^{\prime}}{0}\right)
$$

where $A_{1,2}^{\prime}$ has full row rank while keeping $B_{1,1}$ in echelon form.

At the end of step 2, matrices $A$ and $B$ are represented under the form

$$
P_{1} A_{1} Q_{1}=\left(\begin{array}{c|c}
A_{1,1}^{\prime} & A_{1,2}^{\prime} \\
\hline A_{2} & 0
\end{array}\right) \quad P_{1} B_{1} Q_{1}=\left(\begin{array}{c|c}
B_{1,1}^{\prime} & 0 \\
\hline B_{2} & 0
\end{array}\right)
$$

where

- $A_{1,2}^{\prime}$ has full row rank,

- $\left(\frac{B_{1,1}^{\prime}}{B_{2}}\right)$ has full column rank,

- $\left(\frac{B_{1,1}^{\prime}}{B_{2}}\right)$ and $B_{2}$ are in echelon form.

After steps 1 and 2, we obtain a new pencil of matrices, namely $A_{2}-t B_{2}$. 
Step 3 Starting from $j=2$, repeat the above steps 1 and 2 for the pencil $A_{j}-t B_{j}$ until the $p_{j} \times q_{j}$ matrix $B_{j}$ has full column rank, that is to say until $\operatorname{rank} B_{j}=q_{j}$.

If $B_{j}$ is not a square matrix, then we repeat the above procedure with the transposed pencil $A_{j}^{t}-t B_{j}^{t}$.

At last, we obtain the regular pencil $A^{\prime}-t B^{\prime}$ where $A^{\prime}, B^{\prime}$ are two square matrices and $B^{\prime}$ is invertible.

We are now ready to give our algorithm for solving the curve/surface intersection problem:

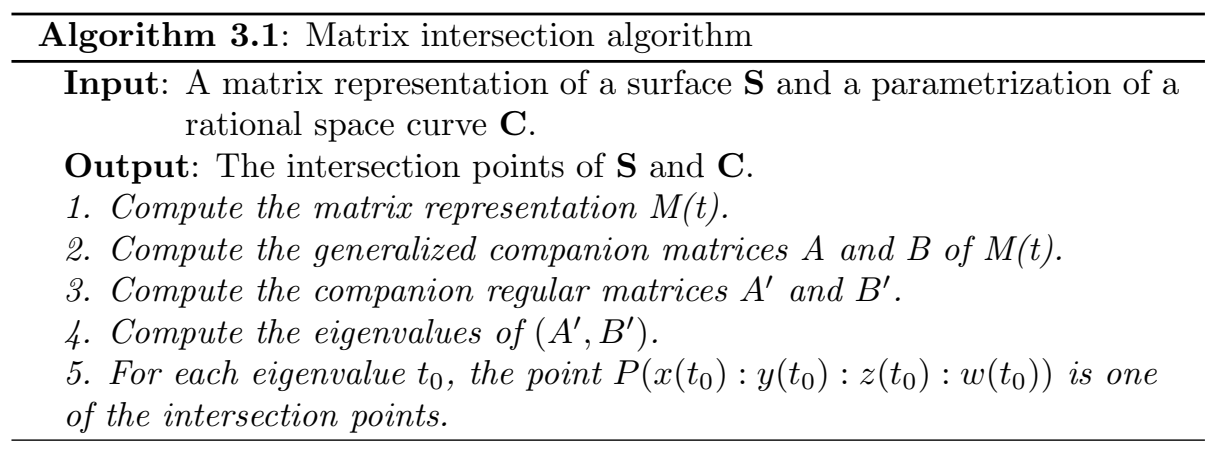

\section{The multiplicity of an intersection point}

In this section, we analyze more precisely the multiplicity of an intersection point and show its correlation with the corresponding eigenvalue multiplicity for the polynomial matrix $M(1, t)$. We assume hereafter, without loss of generality, that the intersection point is at finite distance.

Let $\left(\Delta_{i}(x, y, z, w)\right)_{i=1, \ldots, N}$ be the set of all maximal minors of a representation matrix $M(x, y, z, w)$ of $\mathbf{S}$. By definition, for all $i=1, \ldots, N$ there exists a polynomial $H_{i}(x, y, z, w)$ such that $\Delta_{i}=H_{i} S$ and $\operatorname{gcd}\left(H_{1}, \ldots, H_{N}\right)$ is a nonzero constant in $\mathbb{K}[x, y, z, w]$. Therefore, the zero locus of the polynomials $H_{1}, \ldots, H_{N}, S$ is an algebraic variety $\mathbf{W}$ which is included in $\mathbf{S}$ and which has projective dimension at most one.

Hereafter, we will often abbreviate $x(1, t)$ by $x(t)$ to not overload the text, and will do similarly for the other polynomials $y, z, w$. Let $P=\left(x\left(t_{0}\right): y\left(t_{0}\right)\right.$ : $\left.z\left(t_{0}\right): w\left(t_{0}\right)\right)$ be a point on the parameterized curve $\mathbf{C}$. The intersection multiplicity of $\mathbf{S}$ and $\mathbf{C}$ at $P$ can be defined as

$$
I_{P}=\sum_{t_{i} \text { such that } \Psi\left(t_{i}\right)=P} \operatorname{dim}_{\mathbb{K}}\left(\frac{\mathbb{K}[t]}{S(x(t), y(t), z(t), w(t))}\right)_{\left(t-t_{i}\right)}
$$

assuming w.l.o.g. that $\Psi$ is birational onto $\mathbf{C}$ (by Luröth Theorem [15]) and that all the pre-images of $P$ are at finite distance (that can be achieved by a 
linear change of coordinates). Of course, if $P \in \mathbf{C} \cap \mathbf{S}$ then $I_{P}>0$ and $I_{P}=0$ otherwise. Also, if $P$ is non singular point on $\mathbf{C}$ (recall that the set of singular points on $\mathbf{C}$ is finite) then

$$
I_{P}=\operatorname{dim}_{\mathbb{K}}\left(\frac{\mathbb{K}[t]}{S(x(t), y(t), z(t), w(t))}\right)_{\left(t-t_{0}\right)}
$$

Now, denote by $m_{\lambda}$ the multiplicity of $\lambda$ as a generalized eigenvalue of the matrix $M(t)=M(x(t), \ldots, w(t))$. From the above considerations, it follows that the intersection multiplicity of a point $P=\left(x\left(t_{0}\right): y\left(t_{0}\right): z\left(t_{0}\right): w\left(t_{0}\right)\right) \in \mathbf{C} \cap \mathbf{S}$ such that $P \notin \mathbf{W}$ is exactly the sum of the multiplicity of the corresponding eigenvalues:

$$
I_{P}=\sum_{t_{i} \text { such that } \Psi\left(t_{i}\right)=P} m_{t_{i}}
$$

As already noticed, if $P$ is moreover smooth on $\mathbf{C}$, then $I_{P}=m_{t_{0}}$. Now, if $P \in \mathbf{W} \cap \mathbf{C} \cap \mathbf{S}$, then

$$
I_{P}<\sum_{t_{i} \text { such that } \Psi\left(t_{i}\right)=P} m_{t_{i}}
$$

due to the existence of embedded components (determined by the polynomials $H_{i}$ 's) that come from the matrix representation of $\mathbf{S}$.

Notice that if the surface $\mathbf{S}$ is given by a parameterization which is not birational onto its image, then the matrix representations that we describe in Section 2 actually represent the implicit equation of $\mathbf{S}$ up to a certain power, say $\beta$. In such case, one has similar results regarding the multiplicities of intersection points:

$$
\beta I_{P}=\sum_{t_{i} \text { such that } \Psi\left(t_{i}\right)=P} m_{t_{i}}
$$

If $P$ is smooth on $\mathbf{C}$, then $\beta I_{P}=m_{t_{0}}$ and

$$
\beta I_{P}<\sum_{t_{i} \text { such that } \Psi\left(t_{i}\right)=P} m_{t_{i}}
$$

if $P \in \mathbf{W} \cap \mathbf{C} \cap \mathbf{S}$.

Now, we are going to relate this multiplicity with the multiplicity of the corresponding eigenvalue of the pencil of matrices built in Section 3.3.

With the notations of Section 3.3, we have:

Proposition 5 We have

$$
\operatorname{rank}(A-t B) \text { drops } \Leftrightarrow \operatorname{rank}\left(A^{\prime}-t B^{\prime}\right) \text { drops. }
$$

Proof. It follows from the fact that the Kronecker blocks $L_{i_{1}}, \ldots, L_{i_{s}}, L_{j_{1}}^{t}, \ldots, L_{j_{u}}^{t}$, $\Omega_{k_{1}}, \ldots, \Omega_{k_{v}}$ have all full rank. 
Assume that matrix $M^{t}(t)$ has the Smith form

$$
\left(\begin{array}{cccccc}
a_{r}(t) & 0 & \ldots & \ldots & \ldots & 0 \\
0 & a_{r-1}(t) & 0 & \ldots & \ldots & 0 \\
\vdots & \vdots & \vdots & \vdots & \vdots & \vdots \\
0 & \ldots & \ldots & a_{1}(t) & \ldots & 0 \\
0 & 0 & \ldots & \ldots & \ldots & 0
\end{array}\right)
$$

We set

$$
U(t)=\left(\begin{array}{ccccc}
a_{s}(t) & 0 & \ldots & \ldots & 0 \\
0 & a_{s-1}(t) & 0 & \ldots & 0 \\
\vdots & \vdots & \vdots & \vdots & \vdots \\
0 & \ldots & \ldots & 0 & a_{1}(t)
\end{array}\right)
$$

Notice that $U(t)$ is a square matrix where $a_{1}(t), \ldots, a_{s}(t)$ are monic non constant polynomials.

Proposition 6 The Smith form of the regular pencil $A^{\prime}-t B^{\prime}$ is of the form $\left\{I_{k}, U(t)\right\}$.

Proof. We know that the matrix $A-t B$ has the Kronecker form

$$
\operatorname{diag}\left\{L_{i_{1}}, \ldots, L_{i_{s}}, L_{j_{1}}^{t}, \ldots, L_{j_{u}}^{t}, \Omega_{k_{1}}, \ldots, \Omega_{k_{v}}, A^{\prime}-t B^{\prime}\right\}
$$

and the Smith form (see for instance [20])

$$
\operatorname{diag}\left\{I_{m}, \ldots, I_{m}, D(t)\right\}
$$

On the other hand, we easily see that the Kronecker blocks $L_{k}(t), L_{k}^{t}(t)$ and $\Omega_{k}(t)$ have respectively the Smith form

$$
\begin{gathered}
\left(\begin{array}{ccccc}
1 & 0 & 0 & \ldots & 0 \\
0 & 1 & 0 & \ldots & 0 \\
\vdots & \vdots & \vdots & \vdots & \vdots \\
0 & \ldots & 1 & 0 & 0 \\
0 & 0 & \ldots & 1 & 0
\end{array}\right),\left(\begin{array}{ccccc}
1 & 0 & 0 & \ldots & 0 \\
0 & 1 & 0 & \ldots & 0 \\
\vdots & \vdots & \vdots & \vdots & \vdots \\
0 & \ldots & \ldots & 0 & 1 \\
0 & 0 & \ldots & 0 & 0
\end{array}\right), \\
\left(\begin{array}{ccccc}
1 & 0 & 0 & \ldots & 0 \\
0 & 1 & 0 & \ldots & 0 \\
\vdots & \vdots & \vdots & \vdots & \vdots \\
0 & \ldots & \ldots & 1 & 0 \\
0 & 0 & \ldots & 0 & 1
\end{array}\right) .
\end{gathered}
$$

Therefore, the regular pencil $A^{\prime}-t B^{\prime}$ has the Smith form $\left\{I_{k}, U(t)\right\}$. 
Theorem 3 If $A^{\prime}-t B^{\prime}$ denotes the regular part of a pencil associated to a representation matrix of the intersection between a surface $\mathbf{S}$ and a rational parametric curve $\mathbf{C}$ then the intersection multiplicity of $\mathbf{S}$ and $\mathbf{C}$ at a point $P=\left(x\left(t_{0}\right): y\left(t_{0}\right): z\left(t_{0}\right): w\left(t_{0}\right)\right)$ is equal to the multiplicity of the eigenvalues $\left(A^{\prime}, B^{\prime}\right)$ at $t_{0}$, except in few cases where this multiplicity is strictly bigger.

Proof. Because $M(t)$ is $m \times n$-matrix $(m \leq n)$ representation of the intersection between $\mathbf{S}$ and $\mathbf{C}, M^{t}(t)$ has the Smith form

$$
\left(\begin{array}{cccccc}
a_{m}(t) & 0 & \ldots & \ldots & \ldots & 0 \\
0 & a_{m-1}(t) & 0 & \ldots & \ldots & 0 \\
\vdots & \vdots & \vdots & \vdots & \vdots & \vdots \\
0 & \ldots & \ldots & \ldots & \ldots & a_{1}(t) \\
\vdots & \vdots & \vdots & \vdots & \vdots & \vdots \\
0 & 0 & \ldots & \ldots & \ldots & 0
\end{array}\right)
$$

Let $F(t)=a_{m}(t) a_{m-1}(t) \ldots a_{1}(t)$. By Proposition 6 , we have $F(t)=c \operatorname{det}\left(A^{\prime}-\right.$ $\left.t B^{\prime}\right)$, where $c$ is a nonzero constant. The multiplicity of the eigenvalue of $\left(A^{\prime}, B^{\prime}\right)$ at $t_{0}$ is equal to the multiplicity of the root $t_{0}$ of $F(t)$ and therefore to the multiplicity of $\mathbf{S}$ and $\mathbf{C}$ at a point $P=\left(x\left(t_{0}\right): y\left(t_{0}\right): z\left(t_{0}\right): w\left(t_{0}\right)\right)$, expect in few cases that are described in Section 4.

Remark 2 In the statement of this theorem, the few cases where the multiplicity as an intersection point is strictly less than the multiplicity of the corresponding generalized eigenvalue are exactly the cases where the curve cut out the surface on $\mathbf{W}$, taking again notation of Section 4. It turns out that $\mathbf{W}$ is a closed variety in $\mathbf{S}$ and hence the measure of $\mathbf{W}$ in $\mathbf{S}$ is null. Therefore, these cases have a null probability to happen if the surface and the curve are supposed taken randomly.

\section{$5 \quad$ Examples}

We have implemented our curve/surface intersection algorithm, as well as the matrix representations given in Section 2, in the software Maple. Hereafter, we provide some examples to illustrate it.

Example 1 Let $\mathbf{S}$ be the rational surface which is parametrized by

$$
\phi: \mathbb{P}^{2} \rightarrow \mathbb{P}^{3}:(s: t: u) \mapsto\left(f_{1}: f_{2}: f_{3}: f_{4}\right)
$$

where

$$
f_{1}=s^{3}+t^{2} u, f_{2}=s^{2} t+t^{2} u, f_{3}=s^{3}+t^{3}, f_{4}=s^{2} u+t^{2} u .
$$

We want to compute the intersection of $\mathbf{S}$ and the rational curve $\mathbf{C}$, often called the twisted cubic, given by the parameterization

$$
x(t)=1, y(t)=t, z(t)=t^{2}, w(t)=t^{3} .
$$


First, on computes a matrix representation of $\mathbf{S}$ :

$$
\left(\begin{array}{ccccccc}
0 & 0 & 0 & w-y & 0 & 0 & z-x \\
w & 0 & 0 & x & w-y & 0 & 0 \\
x-y-z & 0 & 0 & -z & 0 & w-y & 0 \\
0 & w & 0 & 0 & x & 0 & -y \\
0 & x-y-z & w & 0 & -z & x & y+z-x \\
0 & 0 & x-y-z & 0 & 0 & -z & 0
\end{array}\right)
$$

$A$ point $P$ at finite distance belongs to the intersection locus of $\mathbf{S}$ and $\mathbf{C}$ if and only if $P=\left(1: t: t^{2}: t^{3}\right)$ and $t$ is one of the generalized eigenvalues of the following matrix $M(t)$ given by

$$
\left(\begin{array}{ccccccc}
0 & 0 & 0 & t^{3}-t & 0 & 0 & t^{2}-1 \\
t^{3} & 0 & 0 & 1 & t^{3}-t & 0 & 0 \\
1-t-t^{2} & 0 & 0 & -t^{2} & 0 & t^{3}-t & 0 \\
0 & t^{3} & 0 & 0 & 1 & 0 & -t \\
0 & 1-t-t^{2} & -t^{3} & 0 & -t^{2} & 1 & t^{2}+t-1 \\
0 & 0 & 1-t-t^{2} & 0 & 0 & -t^{2} & 0
\end{array}\right)
$$

We have $M(t)=M_{3} t^{3}+M_{2} t^{2}+M_{1} t+M_{0}$ where $M_{0}, M_{1}, M_{2}, M_{3}$ are respectively

$$
\begin{gathered}
\left(\begin{array}{ccccccc}
0 & 0 & 0 & 0 & 0 & 0 & -1 \\
0 & 0 & 0 & 1 & 0 & 0 & 0 \\
1 & 0 & 0 & 0 & 0 & 0 & 0 \\
0 & 0 & 0 & 0 & 1 & 0 & 0 \\
0 & 1 & 0 & 0 & 0 & 1 & -1 \\
0 & 0 & 1 & 0 & 0 & 0 & 0
\end{array}\right) \\
\left(\begin{array}{ccccccc}
0 & 0 & 0 & -1 & 0 & 0 & 0 \\
0 & 0 & 0 & 0 & -1 & 0 & 0 \\
-1 & 0 & 0 & 0 & 0 & -1 & 0 \\
0 & 0 & 0 & 0 & 0 & 0 & -1 \\
0 & -1 & 0 & 0 & 0 & 0 & 1 \\
0 & 0 & -1 & 0 & 0 & 0 & 0
\end{array}\right) \\
\left(\begin{array}{ccccccc}
0 & 0 & 0 & 0 & 0 & 0 & 1 \\
0 & 0 & 0 & 0 & 0 & 0 & 0 \\
-1 & 0 & 0 & -1 & 0 & 0 & 0 \\
0 & 0 & 0 & 0 & 0 & 0 & 0 \\
0 & -1 & 0 & 0 & -1 & 0 & 1 \\
0 & 0 & -1 & 0 & 0 & -1 & 0
\end{array}\right) \\
\left(\begin{array}{ccccccc}
0 & 0 & 0 & 1 & 0 & 0 & 0 \\
1 & 0 & 0 & 0 & 1 & 0 & 0 \\
0 & 0 & 0 & 0 & 0 & 1 & 0 \\
0 & 1 & 0 & 0 & 0 & 0 & 0 \\
0 & 0 & 1 & 0 & 0 & 0 & 0 \\
0 & 0 & 0 & 0 & 0 & 0 & 0
\end{array}\right)
\end{gathered}
$$


and the generalized companion matrices of $M(t)$ are

$$
A=\left(\begin{array}{ccc}
0 & I & 0 \\
0 & 0 & I \\
M_{0}^{t} & M_{1}^{t} & M_{2}^{t}
\end{array}\right), B=\left(\begin{array}{ccc}
I & 0 & 0 \\
0 & I & 0 \\
0 & 0 & -M_{3}^{t}
\end{array}\right)
$$

Now, applying the algorithm given in Section 3.3, we find that the regular part of the pencil $A-t B$ is the pencil $A^{\prime}-t B^{\prime}$ where $A^{\prime}$ is given by

$$
\left(\begin{array}{ccccccccccccc}
0 & 0 & 0 & 0 & 0 & 0 & 0 & 0 & 0 & 0 & 0 & 1 & 0 \\
0 & 1 & 0 & 0 & 1 & 0 & 1 & 0 & 0 & 0 & 0 & -1 & 1 \\
0 & 0 & 0 & 0 & 0 & 0 & 0 & 1 & 0 & 0 & 0 & -1 & 0 \\
1 & 0 & 0 & 0 & 0 & 0 & 0 & 0 & 0 & 0 & 0 & 0 & 0 \\
0 & 0 & 0 & 0 & 0 & 0 & 0 & 0 & -1 & 0 & 0 & 0 & -1 \\
0 & 2 & 0 & 0 & 2 & 0 & 2 & 0 & -1 & -1 & -2 & -2 & 1 \\
0 & -1 & 0 & 0 & -1 & 0 & -1 & 0 & 2 & 0 & 1 & 1 & 0 \\
0 & 1 & 0 & 0 & 1 & 0 & 1 & 0 & -1 & -1 & -1 & -1 & 0 \\
0 & 1 & 0 & -1 & 1 & 0 & 1 & 0 & 0 & 0 & -1 & -1 & 1 \\
0 & 0 & 0 & 0 & 0 & -1 & 0 & 0 & 1 & 0 & 0 & 0 & 0 \\
0 & 1 & 0 & 0 & 1 & 0 & 0 & 0 & -1 & 0 & -1 & -1 & 0 \\
0 & 1 & -1 & 0 & 1 & 0 & 1 & 0 & -1 & -1 & -1 & -1 & 0 \\
0 & -1 & 0 & 0 & -2 & 0 & -1 & 0 & 0 & 0 & 1 & 2 & -1
\end{array}\right),
$$

and $B^{\prime}$ is the identity matrix. Then, we compute the following eigenvalues: $t_{1}=1$ with multiplicity $3, t_{2}=-1$ with multiplicity 3 and the roots of the equation $Z^{7}+3 Z^{6}-Z^{5}-Z^{3}+Z^{2}-2 Z+1=0$.

Example $\mathbf{2}$ Let $\mathbf{S}$ be the sphere that we suppose given as the image of the parametrization

$$
\phi: \mathbb{P}^{2} \rightarrow \mathbb{P}^{3}:(s: t: u) \mapsto\left(f_{1}: f_{2}: f_{3}: f_{4}\right)
$$

where

$$
f_{1}=s^{2}+t^{2}+u^{2}, f_{2}=2 s u, f_{3}=2 s t, f_{4}=s^{2}-t^{2}-u^{2}
$$

Let $\mathbf{C}$ be the twisted cubic which is parametrized by

$$
x(t)=1, y(t)=t, z(t)=t^{2}, w(t)=t^{3} .
$$

The computation of a matrix representation of the sphere $\mathbf{S}$ gives

$$
\left(\begin{array}{cccc}
-y & 0 & z & x+w \\
0 & -y & -x+w & -z \\
z & x+w & y & 0
\end{array}\right) .
$$

Now, a point $P$ belongs to the intersection of $\mathbf{S}$ and $\mathbf{C}$ if and only if $P=$ $\left(1: t: t^{2}: t^{3}\right)$ and $t$ is one of the generalized eigenvalues of the matrix

$$
M(t)=\left(\begin{array}{cccc}
-t & 0 & t^{2} & 1+t^{3} \\
0 & -t & -1+t^{3} & -t^{2} \\
t^{2} & 1+t^{3} & t & 0
\end{array}\right) .
$$


As before, we easily compute the eigenvalues and find:

$$
\begin{gathered}
t_{1}=0.7373527056, t_{2}=-0.7373527056, \\
t_{3}=0.5405361044+1.031515287 i, t_{4}=-0.5405361044-1.031515287 i, \\
t_{5}=0.5405361044-1.031515287 i, t_{6}=-0.5405361044+1.031515287 i .
\end{gathered}
$$

All these eigenvalues have multiplicity 1. They all correspond to one intersection point between $\mathbf{S}$ and $\mathbf{C}$ which has multiplicity 1. By Bezout Theorem, we find here all the intersection points between these two algebraic varieties (all of them are at finite distance).

Figure 1: Intersection of the sphere and the twisted cubic, the axis $\mathrm{Oz}$

Example $\mathbf{3}$ As the previous example, let $\mathbf{S}$ be the sphere given be the same parametrization and matrix representation. Here, we want to intersect $\mathbf{S}$ with a simple curve $\mathbf{C}$ : the line parametrized by

$$
x(t)=1, y(t)=0, z(t)=0, w(t)=t .
$$

In this case we have

$$
M(t)=\left(\begin{array}{cccc}
0 & 0 & 0 & t+1 \\
0 & 0 & -1+t & 0 \\
0 & 1+t & 0 & 0
\end{array}\right) .
$$

We proceed as in the previous example and now find two eigenvalues: $t_{1}=-1$ with multiplicity 2 and $t_{2}=+1$ with multiplicity 1 . They correspond to the intersection points $P_{1}(1: 0: 0:-1)$ and $P_{2}(1: 0: 0: 1)$ respectively.

It is interesting to notice that in this case the multiplicity of the eigenvalue $t_{1}$, which is 2, is not equal to the multiplicity of the intersection point $P_{1}$, which is 1. This is due to the fact that the matrix representation of $S$ introduces 
an embedded point, namely $P_{1}$ itself, on the sphere. Indeed, the four maximal minors of the matrix representation of $\mathbf{S}$ are given by

$$
\begin{gathered}
-y\left(-y^{2}+x^{2}-w^{2}-z^{2}\right), z\left(-y^{2}+x^{2}-w^{2}-z^{2}\right), \\
(x+w)\left(-y^{2}+x^{2}-w^{2}-z^{2}\right), 0 .
\end{gathered}
$$

Therefore, the zero locus defined by the equations $x+w, y, z,-y^{2}+x^{2}-w^{2}-z^{2}$, which is nothing but the point $P_{1}$, is an embedded component on the sphere.

\section{Conclusion}

In this paper, we propose a new approach to solve the curve/surface intersection problem in the CAGD. We introduce new matrix based representation of rational surfaces that are allowed to be non square, whereas it was the case for almost all the previous related works. Furthermore, we develop a symbolic/numeric algorithm to manipulate these new representations. We hope to extend, in a near future, our approach for computing the intersections between two parameter-

ized surfaces which is one of the very important problems in Computer Aided Geometric Design.

\section{References}

[1] D. A. Aruliah, R. M. Corless, L. Gonzalez-Vega, and A. Shakoori. Geometric applications of the bezout matrix in the lagrange basis. In Proceedings of the 2007 international workshop on Symbolic-numeric computation, pages 55-64, London, Ontario, Canada, 2007. ACM.

[2] T. Beelen and P. Van Dooren. An improved algorithm for the computation of Kronecker's canonical form of a singular pencil. Linear Algebra Appl., 105:9-65, 1988.

[3] N. Botbol, M. Dohm, and A. Dickenstein. Matrix representations for toric parametrizations. arXiv:0807.4802; to appear in Computer Aided Geometric Design, 2009.

[4] L. Busé and M. Chardin. Implicitizing rational hypersurfaces using approximation complexes. J. Symbolic Comput., 40(4-5):1150-1168, 2005.

[5] L. Busé, M. Chardin, and J. Jouanolou. Torsion of the symmetric algebra and implicitization. Proceedings of the American Mathematical Society, 137(06):1855-1865., Feb. 2009.

[6] L. Busé, D. Cox, and C. D'Andrea. Implicitization of surfaces in $\mathbb{P}^{3}$ in the presence of base points. J. Algebra Appl., 2(2):189-214, 2003.

[7] L. Busé and M. Dohm. Implicitization of bihomogeneous parametrizations of algebraic surfaces via linear syzygies. In ISSAC 2007, pages 69-76. ACM, New York, 2007. 
[8] L. Busé and J.-P. Jouanolou. On the closed image of a rational map and the implicitization problem. J. Algebra, 265(1):312-357, 2003.

[9] L. Busé, H. Khalil, and B. Mourrain. Resultant-based methods for plane curves intersection problems. In Computer algebra in scientific computing, volume 3718 of Lecture Notes in Comput. Sci., pages 75-92. Springer, Berlin, 2005.

[10] D. Cox, R. Goldman, and M. Zhang. On the validity of implicitization by moving quadrics of rational surfaces with no base points. J. Symbolic Comput., 29(3):419-440, 2000.

[11] F. R. Gantmacher. Théorie des matrices. Tome 1: Théorie générale. Traduit du Russe par Ch. Sarthou. Collection Universitaire de Mathématiques, No. 18. Dunod, Paris, 1966.

[12] F. R. Gantmacher. Théorie des matrices. Tome 2: Questions spéciales et applications. Traduit du Russe par Ch. Sarthou. Collection Universitaire de Mathématiques, No. 19. Dunod, Paris, 1966.

[13] G. H. Golub and C. F. Van Loan. Matrix computations. Johns Hopkins Studies in the Mathematical Sciences. Johns Hopkins University Press, Baltimore, MD, third edition, 1996.

[14] A. Khetan and C. D'Andrea. Implicitization of rational surfaces using toric varieties. J. Algebra, 303(2):543-565, 2006.

[15] E. Kunz. Introduction to plane algebraic curves. Translated from the original German by Richard G. Belshoff, 2005.

[16] D. Manocha and J. Canny. A new approach for surface intersection. In Proceedings of the first ACM symposium on Solid modeling foundations and CAD/CAM applications, pages 209-219, Austin, Texas, United States, 1991. ACM.

[17] B. Mourrain. Computing isolated polynomial roots by matrix methods. $J$. of Symbolic Computation, Special Issue on Symbolic-Numeric Algebra for Polynomials, 26(6):715-738, Dec. 1998.

[18] B. Mourrain. Bezoutian and quotient ring structure. J. of Symbolic Computation, 39(3):397-415, 2005.

[19] T. Sederberg and F. Chen. Implicitization using moving curves and surfaces. In Proceedings of SIGGRAPH, volume 29, pages 301-308, 1995.

[20] P. Van Dooren and P. Dewilde. The eigenstructure of an arbitrary polynomial matrix: computational aspects. Linear Algebra Appl., 50:545-579, 1983. 